\title{
Liquid crystalline graphene oxide/PEDOT:PSS self-assembled 3D architecture for binder-free supercapacitor electrodes
}

\section{Md. Monirul Islam, Alfred T. Chidembo, Seyed Hamed Aboutalebi, Dean Cardillo, Hua Kun Liu*, Konstantin Konstantinov * and Shi Xue Dou}

Institute for Superconducting and Electronic Materials, University of Wollongong, North Wollongong, NSW, Australia

\section{Edited by:}

Weitao Zheng, Jilin University, China

Reviewed by:

Ali Qajar, Pennsylvania State

University, USA

Hui Xia, Nanjing University of Science and Technology, China

Anna Donnadio, University of Perugia, Italy

\section{*Correspondence:}

Hua Kun Liu and

Konstantin Konstantinov, Institute for Superconducting and Electronic

Materials, University of Wollongong,

AlIM Facility, Innovation Campus,

North Wollongong, NSW 2522,

Australia

e-mail: hua@uow.edu.au;

konstan@uow.edu.au
Binder-free self-assembled 3D architecture electrodes have been fabricated by a novel convenient method. Liquid crystalline graphene oxide was used as precursor to interact with poly(3,4-ethylene-dioxythiophene):poly(styrenesulfonate) (PEDOT:PSS) in dispersion in order to form a conductive polymer entrapped, self-assembled layer-by-layer structure. This advanced network containing PEDOT:PSS enabled us to ascribe the superior electrochemical properties of particular graphene sheets. This layer-by-layer self-assembled 3D architecture of best performing composite (reduced graphene oxide-PEDOT:PSS 25) showed excellent electrochemical performance of $434 \mathrm{~F} \mathrm{~g}^{-1}$ through chemical treatment. To highlight these advances, we further explored the practicality of the as-prepared electrode by varying the composite material content. An asymmetric supercapacitor device using aqueous electrolyte was also studied of this same composite. The resulting performance from this set up included a specific capacitance of $132 \mathrm{Fg}^{-1}$. Above all, we observed an increase in specific capacitance (19\%) with increase in cycle life emphasizing the excellent stability of this device.

Keywords: liquid crystalline, binder-free, self-assembled, conducting polymer, supercapacitor, PEDOT:PSS

\section{INTRODUCTION}

Supercapacitors are advanced electrochemical devices, which have drawn great attention over the previous decade as a modern energy storage structure with high power density, superior charging/discharging processes, low maintenance costs, and long durability (Simon and Gogotsi, 2008). Formation of an electrical double layer (EDL) between opposite charged electrodes is the basic charge storage mechanism of supercapacitors, which is typically supported by the large specific surface area of carbon materials. A wide range of carbonaceous materials (including graphene) have drawn great attention as electrode materials for supercapacitors (Hao et al., 2013).

Graphene oxide is a promising electrode material with superior physical and promising electrochemical properties (Chen et al., 2013; Tan and Lee, 2013). Facile restacking of reduced graphene oxide sheets through van der Waals interaction results agglomeration and the performances are significantly worse than expected (Liu et al., 2010). Tuning of the dimensions and assembled structures of graphene oxide is a key route to enhance its performance, and among them, three-dimensional (3D) structures have been widely explored (Chen et al., 2011; Choi et al., 2012; Liu et al., 2012; Xu et al., 2013b). Recently, amphiphilic liquid crystalline graphene oxide (LC GO) has been widely reported to introduce a new perspective into self-assembly of graphene materials (Aboutalebi et al., 2011b, 2014b; Kim et al., 2011; Chidembo et al., 2013; Jalili et al., 2013; Maiti et al., 2014; Shen et al., 2014). The amphiphilicity and self-assembly properties are more pronounced in the case where ultra-large GO sheets have been utilized to prepare LC GO dispersions in both water and organic solvents
(Aboutalebi et al., 2011b; Jalili et al., 2013). The application of LC GO dispersion for fabrication enables the formation of a layer-by-layer self-assembled $3 \mathrm{D}$ architecture that leads to better electrochemical properties (Aboutalebi et al., 2011a, 2014a; Jalili et al., 2013). Facile restacking of graphene sheets hinders the otherwise high effective surface area of ultra-large LC GO sheets $\left(2000 \mathrm{~m}^{2} \mathrm{~mL}^{-1}\right)$ (Aboutalebi et al., 2011b). It is difficult, however, to observe the intrinsic capacitance of individual graphene sheets, which determine the observed capacitance of this layer-by-layer self-assembled graphene electrode and also the EDL between electrode-electrolyte interfaces. Different methods and various materials, such as metal oxide nanoparticles, organic molecules, carbon nanorods, and especially, conducting polymers $(\mathrm{CPs})$ have been widely used to fabricate graphene nanocomposites with enhanced properties (Bai et al., 2011; Wu et al., 2012; Tan and Lee, 2013; Xu et al., 2013a). Chemical reduction is one of the best ways to amplify the electrochemical performance of $\mathrm{GO}$, is called reduced graphene oxide ( $\mathrm{rGO}$ ). Among the various reducing agents, ascorbic acid (vitamin C) is an ideal substitute due to its economic feasibility and environmental non-toxicity (Fernández-Merino et al., 2010).

Conducting polymers are promising organic electrode materials for supercapacitor applications with excellent electrical conductivity and facile redox capability. Due to the slow diffusion effect of electrolyte ions, however, CP dominated supercapacitors showed insufficient power performance and volumetric variations during cycling due to failure of the electrode structure caused by fast charge/discharge (Snook et al., 2011; Sun and Shi, 2013). Despite these issues, CPs have become attractive as a secondary 
component in supercapacitor electrodes, such as a surface coating on electric double layer capacitor (EDLC) materials (i.e., graphene) (Wang et al., 2012). Graphene-CP composites provide excellent performance as electrode materials, but they are commercially and environmentally incompatible with the roll-to-roll, large-scale electrode manufacturing processes. Most graphene-CP composites that are applicable in supercapacitors are powdery and need a binder prior to electrode preparation (Li et al., 2013). Binding compounds not only make the procedure more complex but also hinder the electrical conductivity and waste energy. Thus, the most suitable way is to find a way to use the CP in supercapacitors both as a binder and as a capacity enhancer.

Poly(3,4-ethylene-dioxythiophene):poly(styrenesulfonate) (PEDOT:PSS) is a polymer mixture of $p$-type doped conjugated PEDOT polymer, carrying positive charges, and PSS, containing sulfonyl groups to serve as "counter-ions" to PEDOT. Commercially available PEDOT:PSS dry pellets are easily dispersible in aqueous solution and suitable for thin-film formation. PEDOT:PSS also has very good binding capability with metal current collectors. Owing to its very high conductivity, aqueous dispersibility, processing simplicity, and atmospheric stability as a charged organic backbone, PEDOT:PSS has been widely used as a conductive electrode for flexible electronic and optoelectronic devices (Lee et al., 2013; Fan et al., 2014; Yoo et al., 2014). With the advantage of being environmentally benign, PEDOT:PSS can be a very good supercapacitor electrode material candidate compared with other CPs such as polyaniline or polypyrrole. Nevertheless, PEDOT:PSS-rich supercapacitor electrodes have so far exhibited very limited capacitance $\left(<100 \mathrm{~F} \mathrm{~g}^{-1}\right)$, limited power performance, and finite mass loading (Antiohos et al., 2011; Weng and Wu, 2013). As the energy device performance is determined by the electrode active component, it is crucial to engineer hierarchical architectures to provide both binding capability and proper mass loading.

In the present study, we followed a very convenient one-step method to fabricate a layer-by-layer self-assembled 3D architecture of graphene-CP composite for supercapacitor application by using LC GO precursor. After consideration of the environmental issues and atmospheric stability, PEDOT:PSS was chosen as a CP to uniformly coat the liquid crystalline large graphene oxide sheets, while simultaneously maintaining interlayer spacing between the graphene sheets. PEDOT:PSS not only acts as a spacing agent between graphene oxide sheets, but the excess polymer layer plays a vital role as a binding agent, providing satisfactory active material mass loading on the electrode substrate with promising electrochemical performance. Chemical modification of the as-prepared GO-PEDOT:PSS composite electrodes is the key to preserve the large sheet size, even after the reduction of GO. The chemically reduced binder-free GO-PEDOT:PSS composite electrode having less conductive polymer (PEDOT:PSS) content exhibits an enhanced capacitance of $121 \mathrm{~F} \mathrm{~g}^{-1}$ /electrode at a discharge current of $0.25 \mathrm{Ag}^{-1}$ in a real asymmetric supercapacitor (ASC) device. Moreover, all the composite electrodes present outstanding cycling performance with excellent electrical conductivity. By considering the simplicity of fabrication and excellent electrochemical performance with practical composite material mass loading, it is clear that the as-prepared composite possesses great potential as large-scale electrode assembly for supercapacitor applications.

\section{EXPERIMENTAL LC GO SYNTHESIS}

The procedure for the synthesis of graphene oxide liquid crystals is based on our previously reported method (Aboutalebi et al., 2011b; Jalili et al., 2013). Orgacon ${ }^{\mathrm{TM}}$ DRY re-dispersible PEDOT:PSS pellets (produced by Agfa), activated carbon (AC), PVDF, vitamin $\mathrm{C}$, and ethanol were purchased from Sigma-Aldrich and used as supplied.

\section{PREPARATION OF COMPOSITE ELECTRODE}

The preparation of LC GO/PEDOT:PSS composite dispersion and self-assembled 3D architecture electrodes is illustrated in Figure 1. A given amount of PEDOT:PSS was added into $50 \mathrm{~mL}$ of diluted LC GO dispersion in water with a mass ratio of 25,50 , and $75 \%$, with the products denoted as GO-PP 25, GO-PP 50, and GO-PP 75, respectively. PEDOT:PSS was easily dispersed and able to adsorb on GO sheets to form a coating. The polymer-containing dispersion GO-PP 25, 50, and 75 were then dried for 24, 30, and $36 \mathrm{~h}$, respectively, under continuous stirring at room temperature until it formed a viscous paste. The working electrode was prepared by painting the GO-PP composite on titanium sheets $(2 \mathrm{~cm} \times 1 \mathrm{~cm})$ for testing in a three-electrode system, composite material also spread on a circular stainless steel substrate of $15 \mathrm{~mm}$ diameter for testing in an asymmetric device as cathode and allowed to dry at room temperature. AC was mixed with PVDF at a mass ratio of 75:25 to prepare anode for the asymmetric device. Deoxygenation of the GO content was conducted by treating the samples with required amount $(10 \mathrm{~mL})$ of $0.1 \mathrm{M}$ vitamin $\mathrm{C}$ solution at $80^{\circ} \mathrm{C}$ for $12 \mathrm{~h}$. The treated electrodes were washed with ethanol and dried in a vacuum oven. The final loading of the composite material for each electrode was varied from 0.5 to $4 \mathrm{mg} \mathrm{cm}^{-2}$ and thickness ranges from 2 to $18 \mu \mathrm{m}$. Free-standing GO-PP composite paper was cast on a Teflon mold at room temperature. The PEDOT:PSS coated graphene oxide and reduced graphene oxide composites are designated as GO-PP and $\mathrm{rGO}-\mathrm{PP}$, respectively.

The morphology of the composite samples was studied by field emission scanning electron microscopy (FE-SEM) (JEOL JSM7500FA), and high-resolution transmission electron microscopy (HR-TEM) was performed on a JEOL F3000 instrument. Sectioned piece of rGO-PP composite film was mounted on sample holder with carbon tape for FE-SEM analysis. The rGO-PP film was ground lightly with ethanol in a mortar and pestle to disperse it. The grid was then dipped in the suspension, allowed to dry, and examined in the TEM. X-ray diffraction (XRD) experiments were performed using a XRD system (GBC MMA) employing $\mathrm{Cu}$ $\mathrm{K} \alpha$ radiation (X-ray wavelength $\lambda=1.5406 \AA$, with operation at $40 \mathrm{keV}$ and a cathode current of $20 \mathrm{~mA}$ ). Optical absorbance was measured on an ultraviolet-visible (UV-VIS) spectrometer (Shimadzu, UV-3600 UV-VIS-NIR), and Fourier transform infrared (FT-IR) spectra were collected on an AIM-8800 (Shimadzu, Japan) infrared spectrophotometer with the $\mathrm{KBr}$ pellet technique. X-ray photoelectron spectroscopy (XPS, PHOIBOS 100 hemispherical analyzer, and SPECS GmbH) was conducted with pass energy of $26.00 \mathrm{eV}, 45^{\circ}$ take-off angle, and a beam size of $100 \mathrm{~mm}$. The birefringence behavior of the LC GO and LC GO-PEDOT:PSS dispersions was examined by polarized optical microscopy (POM and Leica CTR 6000), operated in transmission mode to examine 


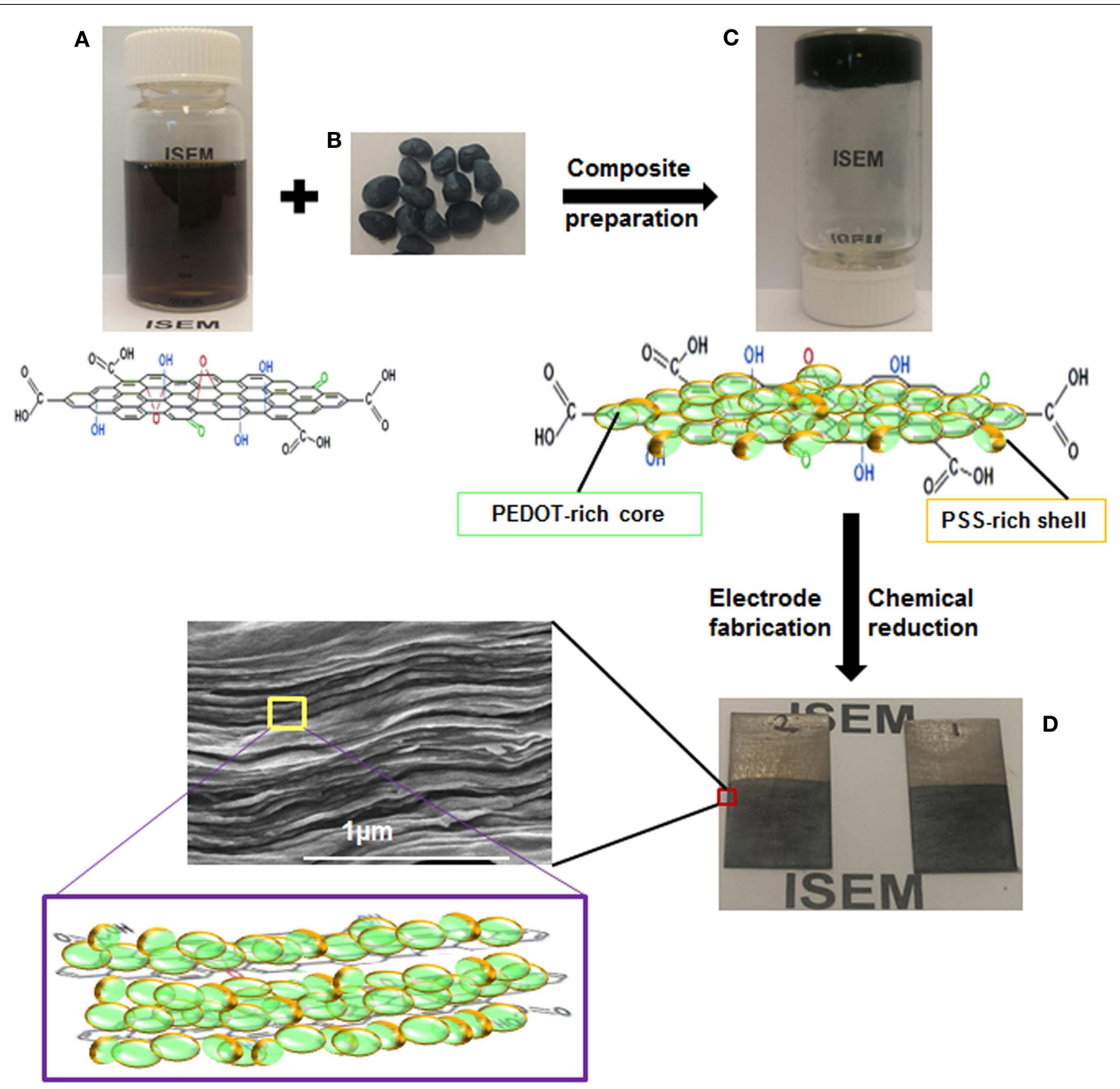

FIGURE 1 | Schematic illustration of preparation of binder-free electrodes: (A) LC GO, (B) PEDOT:PSS, (C) GO-PP composite, and (D) binder-free as-prepared electrodes.

a drop of dispersion on a glass slide. The conductivity of the free-standing composite papers was measured using a four-point probe resistivity system with a linear four-point probe head. Electrochemical experiments were performed on a VMP3 Bio-Logic electrochemical workstation using the three-electrode configuration in a beaker-type cell. An electrolyte solution of $1 \mathrm{M} \mathrm{H}_{2} \mathrm{SO}_{4}$, an $\mathrm{Ag} \mid \mathrm{AgCl}$ reference electrode, and a platinum foil counter electrode were used. An aqueous ASC was assembled in an ECC-Std electrochemical test cell (EL-cell) with two gold current collectors (15 $\mathrm{mm}$ in diameter) and a glass fiber separator.

\section{RESULTS AND DISCUSSION \\ STRUCTURAL CHARACTERIZATION}

Liquid crystalline ultra-large GO sheets are amphiphilic in nature, which therefore makes them an ideal candidate as a water processable material (Aboutalebi et al., 2011a). The basal plane of LC GO is very suitable for holding functional groups of conductive polymer through $\pi-\pi$ interactions. Aggregation of reduced GO sheets is an obstacle to the formation of a homogeneous conductive polymer coating on the reduced GO sheet surface in aqueous solution (Kuilla et al., 2010). Highly dispersible ultra-large LC GO is used to facilitate the interaction with CPs prior to the formation of the composite and chemical reduction of the as-prepared composite material to resolve the aggregation issue. PEDOT:PSS pellets were homogeneously dispersed in LC GO dispersion as an aqueous medium containing GO sheets with their surfaces exposed for easy interaction. GO sheets with PEDOT:PSS coating showed excellent dispersion capability, and their liquid crystalline properties remained stable. Representative POM micrographs of the composite dispersion in Figure 2B clearly show the birefringent lyotropic LC behavior of PEDOT:PSS interacting with GO in water. The lyotropic nematic liquid crystalline properties of the GO dispersion became more obvious with increasing polymer content (see Figure S1 in Supplementary Material).

The liquid crystalline properties of GO provide unique opportunities for the production of a self-assembled, fully ordered, and 

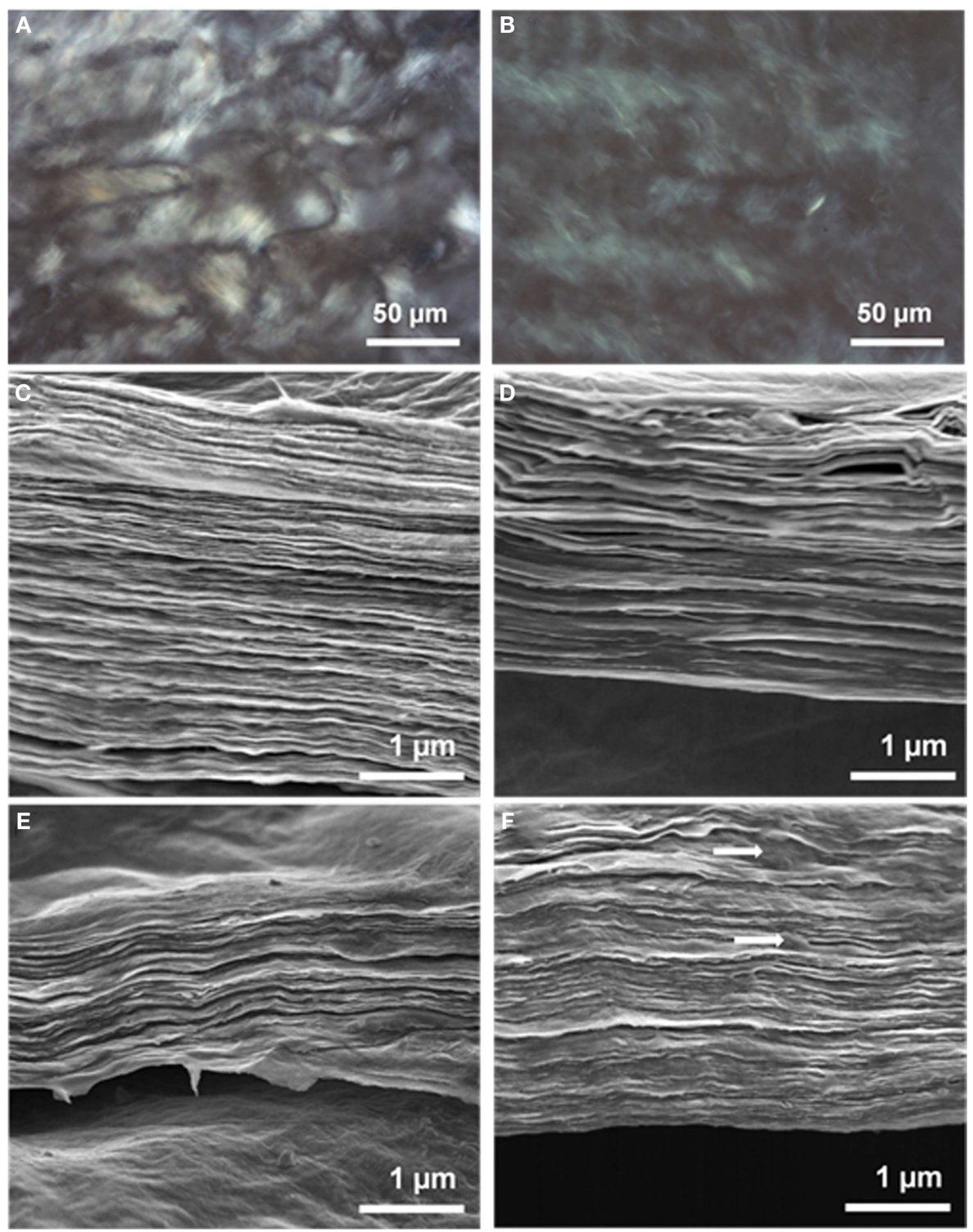

FIGURE 2 | Representative polarized optical microscope images of (A) LC GO and (B) GO-PP 25 at a concentration of $2.5 \mathrm{mg} \mathrm{mL}^{-1}$. FE-SEM micrographs of dried composites: (C) GO, (D) GO-PP 25, (E) GO-PP 50, and (F) GO-PP 75, with the arrows marking the PEDOT:PSS overlap.

novel composite architecture with free-standing and flexible film formation. Figure S2 in Supplementary Material shows a piece of $25 \%$ PEDOT:PSS composite paper $16 \mathrm{~cm} \times 12 \mathrm{~cm}$ in size. The cross-sectional FE-SEM images of different PEDOT:PSS composite papers in Figure 2 show the ordered multi-layer structure of the PEDOT:PSS coated thin graphene sheets. Interaction between the GO sheets and the entrapped polymer between the layers leads to increasing GO sheet thickness with increasing PEDOT:PSS content from 25 to $75 \%$. GO-PP 25 composite shows almost the same architecture and sheet thickness as GO, but the higher polymer content composites (GO-PP 75) show ordered assembly with polymer overlap from the composite interior and form a compact structure.
The self-assembled, multi-layer 3D architecture is maintained even after chemical reduction. Figure S3 in Supplementary Material presents high-resolution cross-sectional FE-SEM images of the chemically treated composites. The increasing sheet thickness of the PEDOT:PSS coated GO can also be detected from these images. The surface morphology of the GO sheets has also changed due to the polymer coating. A small amount of PEDOT:PSS (25\%) attaches itself to the GO sheets uniformly and makes the sheet surface smoother than that of bare GO, but with increasing PEDOT:PSS content, localized PEDOT:PSS agglomeration from the aqueous dispersion may occur, appearing as nanowhiskers on the GO surface (Figures 3C,D). Figure 3 also contains TEM images of GO and GO-PP 25 composite to illustrate the 

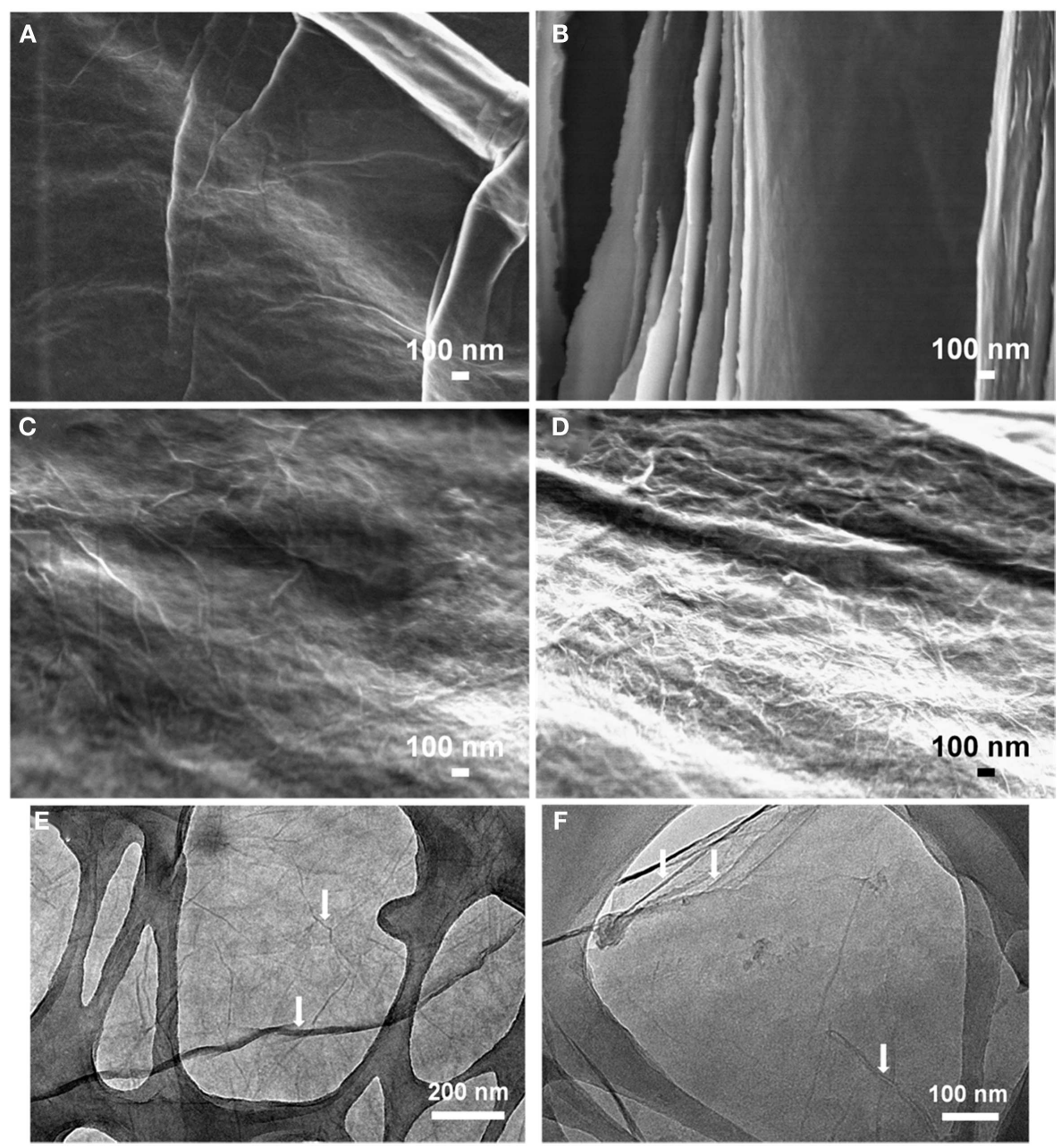

FIGURE 3 | Field emission scanning electron microscopy images showing the surface morphology of (A) GO, (B) GO-PP 25, (C) GO-PP 50, and (D) GO-PP 75. Representative TEM images of (E) GO film and (F) GO-PP 25 composite.

structural morphology. The layer-by-layer self-assembled structure is clearly evident from the homogeneous PEDOT:PSS among the GO sheets. The characteristic wrinkles in the GO sheets in Figure $3 \mathrm{E}$ have almost disappeared with the low mass loading of PEDOT:PSS in the composite paper (Figure 3F). The flexible GO-PEDOT:PSS composite paper exhibits good electrical conductivity of $72 \mathrm{~S} \mathrm{~cm}^{-1}$ for the $25 \%$ PEDOT:PSS composite, but chemical treatment increases the conductivity to $343 \mathrm{~S} \mathrm{~cm}^{-1}$. In the chemical reduction process, like different solvents (Ouyang et al., 2004) ascorbic acid solution most likely results in the conversion of the PEDOT chain from a benzoid to quinoid structure and induces significant conductivity enhancement in the composite films.

The PEDOT:PSS between the composite layers also increases the layer-to-layer distance ( $d$-spacing) compared to GO, as illustrated in Figure 4A. The XRD pattern of the as-prepared GO shows a distinct peak at $10.83^{\circ}$, corresponding to a $d$-spacing of about $8.17 \AA$ for typical GO sheets (Aboutalebi et al., 2011a). The introduction of $25 \%$ PEDOT:PSS forms a very thin layer on the GO sheets and downshifts the peak to $10.46^{\circ}$, which is analogous to a $d$-spacing of about $8.450 \AA$, demonstrating the very thin nature of the polymer layers during the formation of the self-assembled ordered structure. The same trend is observed with increasing amounts of PEDOT:PSS. In the composite with 50\% PEDOT:PSS, the peak is shifted to $9.90^{\circ}$, indicating a $d$-spacing of $8.927 \AA$, but the excess polymer content interacts with itself and results in an amorphous composite (Ouyang et al., 2004), which reduces the graphene oxide characteristic peak and makes it difficult to determine the interlayer distance in the 75\% PEDOT:PSS composite. The XPS survey spectra of GO and GO-PP composite in Figure 4B show the presence of $\mathrm{C} 1 \mathrm{~s}$ and $\mathrm{O}$ 1s signals, which resemble the typical S 2p signal of PEDOT:PSS, indicating that PEDOT:PSS had been coated on the GO sheets uniformly. The high-resolution C 1s XPS spectrum (Figure 4C) of the GO-PEDOT:PSS composite shows four peak components with binding energies at about 284.4, 285.5, 286.8, and $287.8 \mathrm{eV}$, which are attributed to $\mathrm{sp}^{2}$-hybridized carbon, $\mathrm{sp}^{3}$-hybridized carbon, $\mathrm{C}-\mathrm{S}, \mathrm{C}-\mathrm{O}, \mathrm{C}=\mathrm{O}$, and $\mathrm{O}-\mathrm{C}=\mathrm{O}$ species, respectively (Jo et al., 2011). 

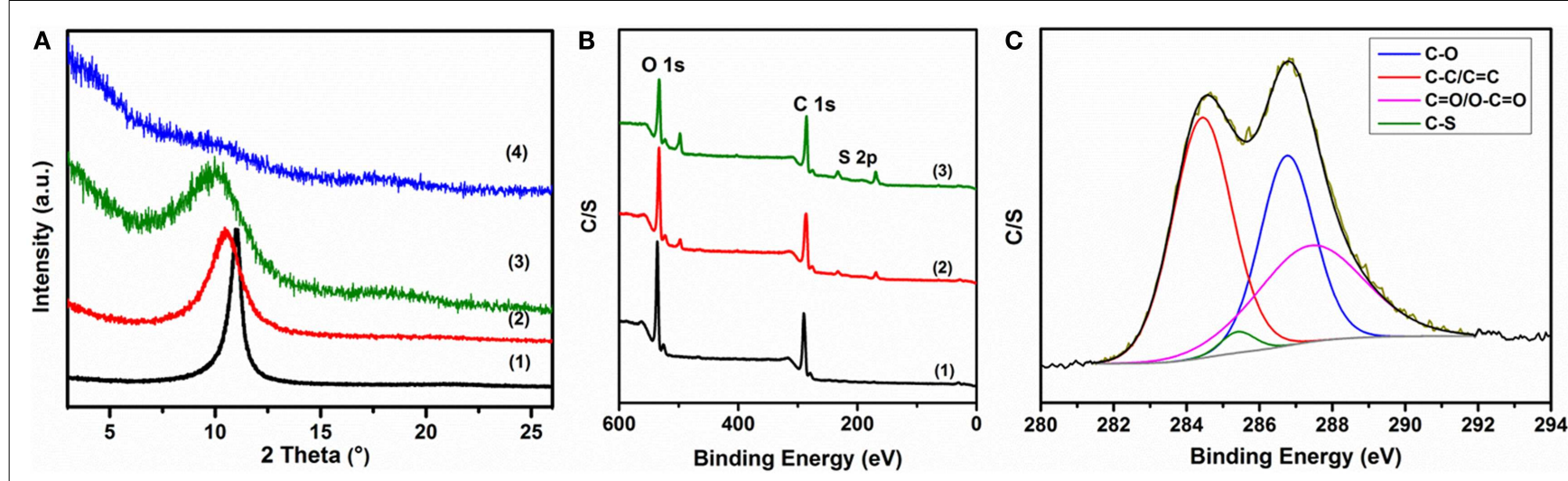

FIGURE 4 | Structural characterization of composites: (A) comparison of XRD patterns of as-prepared composites: (1) GO, (2) GO-PP 25, (3) GO-PP 50, and (4) GO-PP 75; (B) XPS survey spectra of (1) GO, (2) GO-PP 25, and (3) rGO-PP 25 composites; and (C) fitted GO-PP 25 composite core level spectrum in the carbon region.
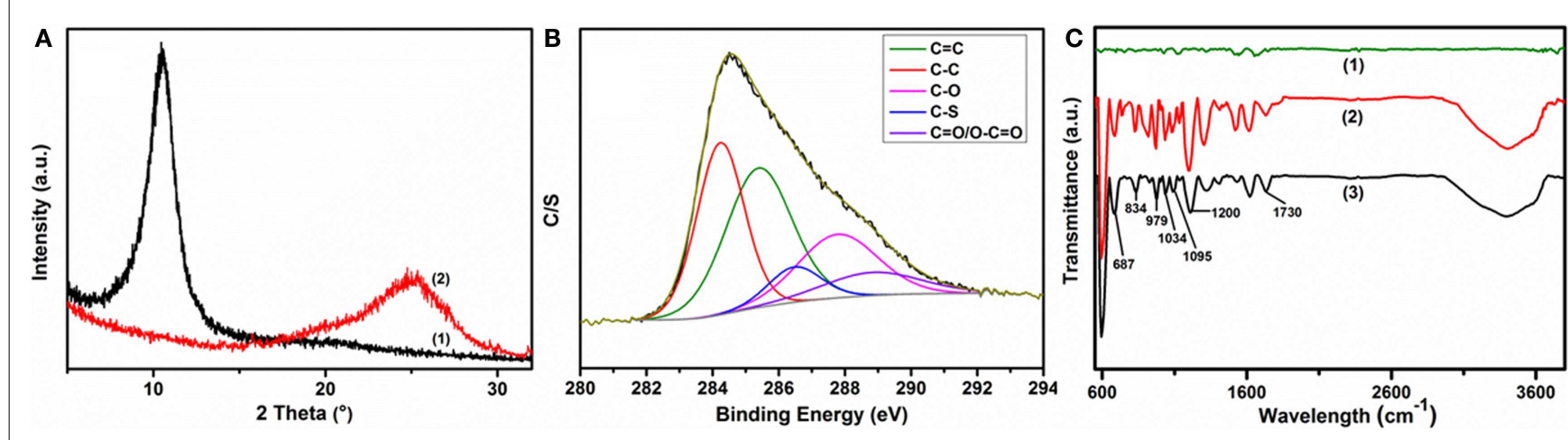

FIGURE 5 | Chemical reduction effects on as-prepared composite materials: (A) change in XRD patterns of (1) GO-PP 25 and (2) rGO-PP 25 composites; (B) XPS core level spectra of rGO-PP 25 composite in the carbon region; and (C) comparative FT-IR spectra of (1) rGO, (2) GO-PP 25, and (3) rGO-PP 25 composites.

\section{FABRICATION OF ELECTRODE AND CHEMICAL REDUCTION}

A one-step easy fabrication method has been used to prepare electrodes with layer-by-layer self-assembled 3D architecture, where the GO sheets are homogeneously coated with a PEDOT:PSS layer. Interlayer PEDOT:PSS not only facilitates electrochemical access to the GO sheet surfaces but also acts as a binder to promote the attachment of the electrode materials to the current collector, even after chemical treatment, whereas GO and PEDOT:PSS are unable to exhibit this property themselves (Figure S4 in Supplementary Material). Chemical treatment of the as-prepared electrodes by dipping them into a reducing agent solution has been chosen as a convenient way to reduce the GO content in the GO-PP composite. This directly pasted, binder-free, self-assembled electrode served as a robust platform to prepare graphene- $\mathrm{CP}$ composite electrodes at moderate temperature, by avoiding highly toxic reducing agents (hydrazine) and minimizing the risk of introducing heteroatom into the electrode materials (Fernández-Merino et al., 2010; Zhang et al., 2010).

Distinct changes were observed in the XRD patterns during the chemical processing (Figure 5A). The peak position of
25\% PEDOT:PSS containing GO composite at $10.46^{\circ}(8.45 \AA)$ was shifted to a higher angle of $24.74^{\circ}(3.595 \AA)$, close to the interlayer spacing of graphite of $3.34 \AA$ at $26.6^{\circ}$, due to the remarkable loss of functional groups on the GO sheets during the chemical reduction. The S 2p core level spectra of rGO-PP 25 composite suggest a uniform coating and stability of PEDOT:PSS on the graphene sheets (Figure S5 in Supplementary Material). The lower-energy feature of the $\mathrm{S} 2 \mathrm{p}$ spectrum, near $165 \mathrm{eV}$, can be assigned to the sulfur atoms in PEDOT, and the higher energy spectrum near $169 \mathrm{eV}$ resembles that of the sulfur atoms in PSS (Kim et al., 2012) in spectrum (3) in Figure 3B, revealing the residual oxygenated groups on graphene after chemical reduction, while the $S 2 p$ signal intensity in the survey spectrum [Figure 3B, spectrum (3)] indicates the increased ratio of sulfur to carbon or oxygen as an effect of chemical reduction. Moreover, the core level C 1s spectrum of rGO-PP 25 composite demonstrates that the proportion of epoxy/hydroxyls and carboxylate groups decreases remarkably relative to carbon-carbon or carbon-sulfur groups, implying an efficient reduction of graphene oxide through chemical treatment. 
Figure 5C presents the FT-IR spectrum of GO-PP 25 along with those of rGO and rGO-PP 25 composite. Compared with rGO and GO-PP 25, rGO-PP 25 composite shows characteristic peaks of PEDOT:PSS [Figure 5C, spectrum (3)]. The peaks at 979, 834 , and $687 \mathrm{~cm}^{-1}$ can be assigned to the C-S bond of the thiophene ring in PEDOT. The bands at 1200,1095 , and $1034 \mathrm{~cm}^{-1}$ are ascribed to stretching modes of the alkylenedioxy group (Sun et al., 2013). Vibrations below $600 \mathrm{~cm}^{-1}$, the $\mathrm{S}=\mathrm{O}$ vibration near $1200 \mathrm{~cm}^{-1}$, and the $\mathrm{O}-\mathrm{S}-\mathrm{O}$ signal at $1034 \mathrm{~cm}^{-1}$ are identical to those for the sulfonic acid group of the PSS chain (Friedel et al., 2009). The following results have definitely proved the effective reduction of GO and the presence of stable PEDOT:PSS layers in the 3D architecture of the composite. The stability of the PEDOT:PSS after chemical treatment at $80^{\circ} \mathrm{C}$ is also supported by the UV-VIS spectra in Figure S6 in Supplementary Material. The PEDOT:PSS incorporated, self-mediated 3D architecture platform of rGO-PP composite paper showed satisfactory mechanical strength compared to free-standing rGO paper (Table S1 in Supplementary Material). A further increase in conductive polymer content between rGO layers decreases the composite flexibility and mechanical strength.

\section{ELECTROCHEMICAL PERFORMANCE}

Cyclic voltammetry $(\mathrm{CV})$ was used to determine the effects of reduced graphene oxide in the $\mathrm{rGO}-\mathrm{PP}$ composite, as shown in Figure 6A. An increase in the area of the CV suggests that the reduction of GO has a massive impact on the overall specific capacitance of the composite. From the same plot, the EDLC and pseudocapacitive contributions from the rGO and PEDOT:PSS are evidenced by the large current separation and distorted rectangular shape of the CV. Although CPs are well known to be pseudocapacitive in nature, thereby giving rise to peaks in the $\mathrm{CV}$, it is expedient to assume that the overall pseudocapacitive effect arises from both the rGO and the PEDOT:PSS. Several groups have reported high-performance graphene oxide-PEDOT:PSS composite electrodes with a very minute mass loading (Weng and $\mathrm{Wu}$, 2013) listed in Table 1, which is not practical for commercial applications. Small masses also exaggerate the performance of such electrodes (Stoller and Ruoff, 2010). We therefore set out to determine the optimum mass loading for the best and consistent performance and compared our results to those reported literature of similar composites, as shown in Table $\mathbf{1}$.

From the results in Figure 6B, we observe the effects of mass loading on the overall specific capacitance. Prepared electrode with minute mass $\left(0.5 \mathrm{mg} \mathrm{cm}^{-2}\right)$ showed extraordinary performance of $434 \mathrm{Fg}^{-1}$. With an increase of active material (rGO-PP composites) content, specific capacitance $\left(C_{s}\right)$ starts to decrease, but at higher mass loading of $2.15-4 \mathrm{mg} \mathrm{cm}^{-2}$ an overall and consistent performance of around $246 \mathrm{~F} \mathrm{~g}^{-1}$ was achieved by using the following equation.

$$
C_{\mathrm{s}}=\frac{1}{\operatorname{mv}\left(V_{a}-V_{c}\right)} \int_{0}^{0.8} I(V) \mathrm{dV}
$$

where $m$ is the mass of active material (rGO-PP) (g), $v$ the scan rate $\left(\mathrm{mV} \mathrm{s}^{-1}\right), I$ the current, and $V$ the voltage, while $\left(V_{a} \times V_{c}\right)$ represents the potential window and the integral area under the
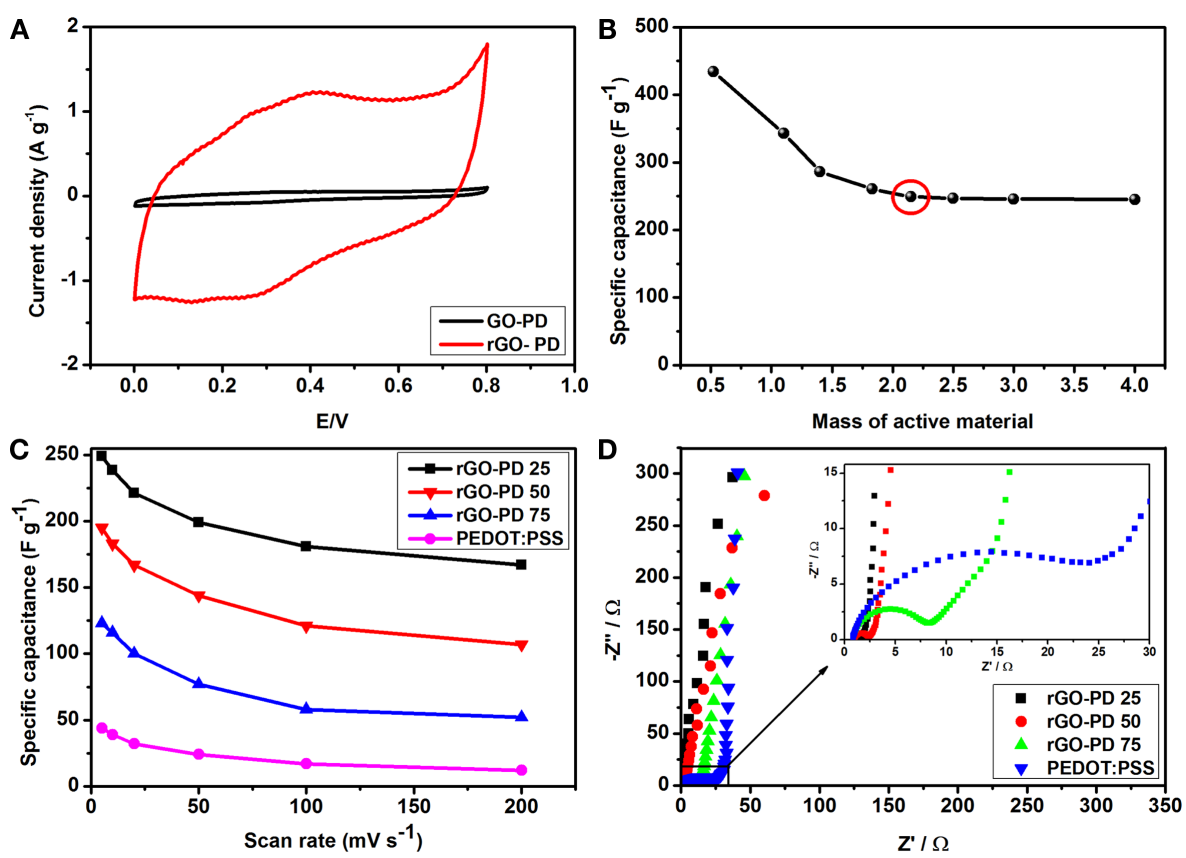

FIGURE 6 | Electrochemical performance of rGO-PP 25 composite in a three-electrode system: (A) cyclic voltammograms of the composite electrodes before and after chemical reduction, (B) specific capacitance of rGO-PP 25 composite electrodes with different mass loading at 5 mV $\mathrm{s}^{-1}$, marked with the minimum active material mass for further electrochemical characterization, (C) variation of specific capacitance of different composites including PEDOT:PSS with scan rates, and (D) Nyquist plots of different composite electrodes. The inset shows the corresponding magnified high frequency region. 
Table 1 | Graphene/CNTs-PEDOT:PSS based composites used as supercapacitor electrode material.

\begin{tabular}{llccc}
\hline Composite & Method of preparation & Mass loading $\mathbf{~ m g ~ c m}-\mathbf{2}$ & Specific capacitance F $\mathbf{g}^{\mathbf{- 1}}$ & Reference \\
\hline rGO-PEDOT:PSS & Solution cast & 0.52 & 434 & Current study \\
rGO-PEDOT:PSS & Solution cast & 2.15 & 249 & Current study \\
GO-PEDOT:PSS-CNT & Solution cast & 0.3 & 365 & Weng and Wu (2013) \\
Graphene/PEDOT & Microwave-assisted & 0.03 & 270 & Sun et al. (2013) \\
rGO-PEDOT & In situ polymerization & 2 & 108 & Zhang and Zhao (2012) \\
PEDOT:PSS-SWCNT & Mixing by sonication & 4 & 133 & Antiohos et al. (2011) \\
\hline
\end{tabular}

cyclic voltammograms. Our obtained values of $C_{\mathrm{s}}$ for higher mass electrodes are better than the electrodes of similar composition and mass loading previously reported (Table 1). We attribute these high values for performance at variable mass loadings in our study, compared to the literature data (Table 1) to the influence of the differing morphology of our ultra-large rGO sheets separated by PEDO:PSS. From Figure 6B, we demonstrate that the specific capacitance is not affected by further increasing the quantity of active material over $2.15 \mathrm{mg} \mathrm{cm}^{-2}$. To achieve more realistic specific capacitance of our composite electrode as suggested by Stoller and Ruoff (2010), we selected a mass loading of over $2.15 \mathrm{mg} \mathrm{cm}^{-2}$ as an appropriate quantity (Figure 6B) to carry out further tests to determine the effects of the scan rate on the electrode materials. This information points directly to the power capability of an electrode. From Figure 6C, a reduction in the specific capacitance with increasing scan rate was observed. This is due to the fact that at high scan rates, electrolyte ions do not get sufficient time to occupy the available sites for charge storage. Even at $200 \mathrm{mV} \mathrm{s}^{-1}$, rGO-PP 25 still produced a specific capacitance of $167 \mathrm{~F} \mathrm{~g}^{-1}$. However, an increase in PEDOT:PSS content should enhance the specific capacitance due to its faradic contribution, but diffusion effects of the electrolyte ions are slower with an increase in PEDOT:PSS content and show lower capacitance (Zhang and Zhao, 2012). This shows that the synergy between the rGO and PEDOT:PSS results in a robust composite that stores more charge than rGO and PEDOT PSS individually.

Figure 6D shows the electrochemical impedance spectroscopy (EIS) plot obtained for the composite electrode over the frequency range from $10 \mathrm{kHz}$ to $10 \mathrm{mHz}$. The Nyquist plots of different rGO-PP composites and PEDOT:PSS show a typical high frequency semicircle, signifying charge transfer resistance, and an almost vertical plot at low frequency where the electrodes are dominated by purely capacitive behavior. From the inset in Figure 6D, charge transfer resistance, $R_{\mathrm{ct}}$, values of $0.76,1.34$, and $7.13 \Omega$ were recorded for rGO-PP 25, rGO-PP 50, and rGO-PP 75, respectively. The analyses show that the good electrical conductivity and ionic diffusion resulted in the high-performance of the rGO-PP 25 composite as electrode material for supercapacitor electrode. We envisage that a lesser amount of conductive polymer provides better connection between the graphene layers to facilitate improved electrical conductivity.

Unlike many other reported graphene-CP composites (Zhang and Zhao, 2012; Weng and Wu, 2013) chemically reduced GOPEDOT:PSS composite showed a very interesting phenomenon and excellent performance, as it exhibited noticeably increased specific capacitance with increasing cycle number. The electrodes made from rGO-PP 25 composite shows a steady increase in capacitance of 19.6\% after 2000 cycles (Figure S7 in Supplementary Material), possibly due to the depletion of residual oxygenated groups on graphene sheets in Figure 5B, causing the material to become more electroactive. This interesting characteristic may be due to the redox effect of PEDOT:PSS on the partial electrochemical reduction of GO and significantly improves the material's cycle life.

\section{ASYMMETRIC SUPERCAPACITOR}

To assess the feasibility of PEDOT:PSS coated graphene composite in a practical system, we assembled an ASC by following best practice method (Stoller and Ruoff, 2010; Tang et al., 2013). The best performing rGO-PP 25 as cathode, together with $\mathrm{AC}$ as the anode material, and tested the supercapacitor in a potential window of $0-1 \mathrm{~V}$. Figure 7A presents the almost rectangular cyclic voltammograms resulting from the combination of rGO-PP 25 composite and $\mathrm{AC}$ as expected for ideal capacitors, even at the high scan rate of $100 \mathrm{mV} \mathrm{s}^{-1}$. The slight decrease in capacitance as the scan rate was increased (Figure 7B), while maintaining the rectangular shape, indicates desirable fast charge/discharge properties for power devices.

A number of factors play influential roles in determining the final performance of a material in supercapacitor device. In this study, we have only focused on the performance of the as-prepared composite electrode. In order to do so, the specific capacitance $\left(C_{\mathrm{s}}\right)$ per unit mass of one electrode ( $\left.\mathrm{rGO}-\mathrm{PP} 25\right)$ in the ASC device was evaluated from CV by applying Eq. 1 and from the charge/discharge (CD) method by applying Eq. 2 (Stoller and Ruoff, 2010; Zhang et al., 2011).

$$
C_{\mathrm{s}}=4 \times \frac{I \times t}{V \times m \pm}=4 \times C_{T}
$$

where $I$ is the current in amperes, $t$ the discharge time in seconds, $V$ the voltage, $m_{ \pm}$the total mass of the positive and negative electrodes in grams and $C_{T}$ is the specific capacitance of the ASC cell. As we have focused on the performance of our as-prepared rGOPP composite electrode in the device, a factor of 4 was used to translate the specific capacitance of the ASC into that of the single rGO-PP 25 electrode. Maximum specific capacitances $\left(C_{s}\right)$ of 139 and $121 \mathrm{~F} \mathrm{~g}^{-1}$ of the rGO-PP 25 composite electrode in the ASC devices were obtained from $\mathrm{CV}$ and $\mathrm{CD}$, respectively. Alternating current penetrates into the bulk of the electrode material with some difficulty, which can cause lower $C_{\mathrm{s}}$ values to be obtained from $\mathrm{CD}$ than those obtained through $\mathrm{CV}$. As mentioned earlier, 

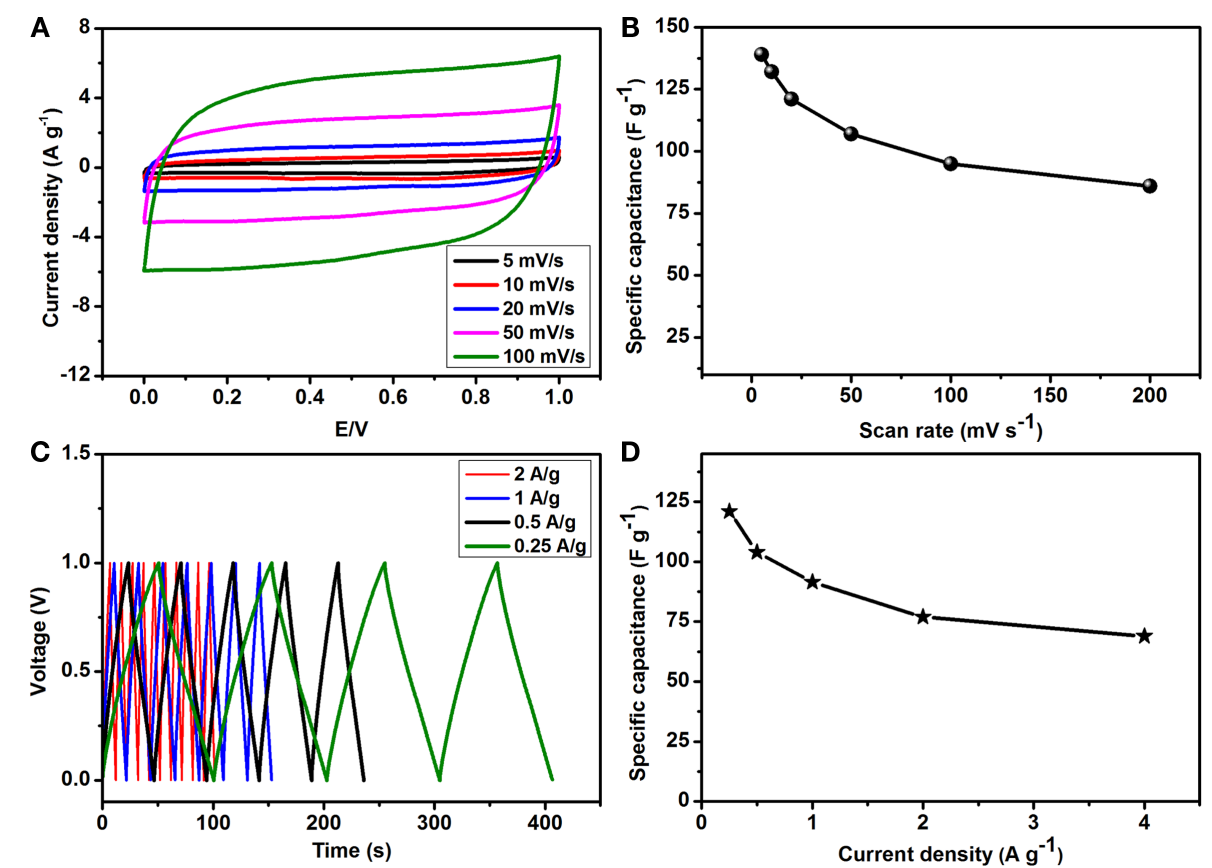

FIGURE 7 | Electrochemical performance of the as-prepared rGO-PP 25 composite in an asymmetric supercapacitor device: (A) effect of scan rates on the specific capacitance $\left(C_{\mathrm{s}}\right)$, (B) variation of specific capacitance at different scan rates, $(C)$ charge/discharge profiles at different current densities, and (D) variation of specific capacitance $\left(C_{\mathrm{s}}\right)$ at different current densities

the combined contribution of constituent materials in the composite from pseudocapacitance and EDLC provides these high capacitances. Figure 7C shows CD profiles obtained at different current densities that exhibit symmetrical linear charging and discharging slopes, implying a rapid $I-V$ response, excellent electrochemical reversibility, and high coulombic efficiency. At higher current densities, specific capacitances of 121, 104, 91, 77, and $69 \mathrm{Fg}^{-1}$ at $0.25,0.5,1,2$, and $4 \mathrm{Ag}^{-1}$ were obtained, as shown in Figure 7D.

Perhaps the most important parameters for any practical supercapacitor device are the energy and power densities. The specific energy (E) and power (P) density of the rGO-PP 25 composite electrode in an ASC device are calculated from the galvanostatic data using the following equations (Zhang et al., 2011) and presented in Table S2 in Supplementary Material (EIS).

$$
\begin{aligned}
& E=\frac{1}{2} C_{\mathrm{s}}(\Delta V)^{2} / 3.6 \\
& P=\frac{E}{t}
\end{aligned}
$$

where $C_{s}, \Delta V$, and $t$ are the specific capacitance of composite electrode in the ASC device, potential window (V), and discharge time (s), respectively. Due to the synergy between the constituent materials in the composite, specific energy density of $16.14 \mathrm{Wh} \mathrm{kg}^{-1}$ and a power density of $1185 \mathrm{~W} \mathrm{~kg}^{-1}$ were achieved for the composite electrode in the assembled ASC device at $0.25 \mathrm{~A} \mathrm{~g}^{-1}$. Including higher mass loading (over $2.15 \mathrm{mg} \mathrm{cm}^{-2}$ ) such a better specific energy density is ideal for supercapacitors, as the challenge for these devices is to store as much energy as batteries. It is not surprising that the power density for this device is also promising, as is characteristic of supercapacitors. Moreover, the energy and power density of rGO-PP 25 composite electrode in an ASC device can be further increased by combining with different anode materials to optimize its performance. With the excellent interconnectivity between the components of the final electrode composite, the ions are able to penetrate throughout the whole porosity of the active material and provide fast charge transfer. For this reason, Nyquist plot in Figure 8A, there is a very small semicircle indicating low charge transfer resistance $\left(R_{\mathrm{ct}}\right)$, while a near vertical line was detected at lower frequencies. A Bode plot (Figure 8B) was plotted from the Nyquist plot to show the maximum phase angle of $-82^{\circ}$, which is close to the phase angle of $-90^{\circ}$ for an ideal supercapacitor.

Long cycle life is a prominent characteristic of supercapacitor electrode materials in practical application. To determine the longevity and study the change in capacitance of the asymmetric device, we tested the performance over 2000 cycles at $50 \mathrm{mV} \mathrm{s}^{-1}$, and the results are plotted in Figure 8C. No substantial changes were observed in the device capacitance, and the internal area of the cyclic voltammograms (Figure 8C) becomes even more rectangular, which is ideal for practical supercapacitors. Interfacial contact between PEDOT:PSS and graphene not only prevents the agglomeration and restacking of graphene sheets but also enhances the electrochemical activity of the composite as an improved EDLC.

Thus, copious electrochemical analysis suggests that the rGOPP composite electrode, which is also easily scaled-up, exhibits 

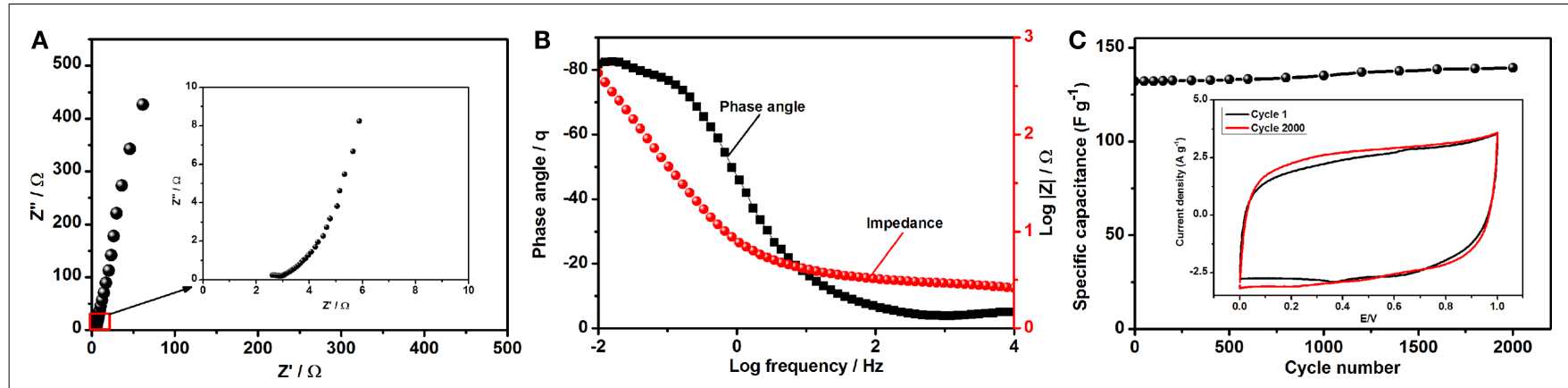

FIGURE 8 | Electrochemical behavior of the assembled asymmetric supercapacitor device: (A) Nyquist plot of the asymmetric supercapacitor, with the inset showing an enlargement of the indicated region; (B) Bode plot suggesting maximum phase angle; and (C) cycle life of the assembled asymmetric super capacitor over 2000 cycles at $50 \mathrm{mV} \mathrm{s}^{-1}$, with the inset showing CV curves for cycles 1 and 2000.

promising specific capacitance with a synergistic effect between the graphene and the PEDOT:PSS. Firstly, the aligned $p$-type PEDOT and $n$-type PSS chains facilitate ion diffusion from the electrolyte to each layer of graphene sheet, making complete use of the active material. In addition, the large surface area of the ultra-large graphene sheets promotes faster electron transfer. Due to this, decreased ion diffusion and charge transfer resistance lead to the improved specific capacitance. Moreover, with the help of graphene sheets as a platform for PEDOT:PSS chains, the composite can withstand the strain relaxation and mechanical deformation from cycling, preventing the electrode material from seriously swelling and shrinking during the electrochemical processes. Therefore, the full interaction of PEDOT:PSS with the graphene sheets resulted in improved capacitive performance of the composite and better cycling stability.

\section{CONCLUSION}

The direct interaction between PEDOT:PSS and LC GO sheets leads to the formation of layer-by-layer self-assembled 3D architecture composite. Interlayer conductive PEDOT:PSS enable this composite as a high quality, innovative, binder-free electrode material through one-step reduction process. Application of ascorbic acid as a reducing agent defuses environment concerns arising from highly toxic reducing agents. The easily scaled-up preparation and chemical reduction are the most practical merits of this present method. By efficiently applying the synergistic effects of the constituent materials, the rGO-PEDOT:PSS composite (rGO-PP 25) as supercapacitor electrode shows, greatly intensified specific capacitance $\left(249 \mathrm{~F} \mathrm{~g}^{-1}\right)$ with appropriate mass loading (over $2.15 \mathrm{mg} \mathrm{cm}^{-2}$ ), and better cycle life than pure graphene and PEDOT:PSS individually. Considering the potential practical applications in portable energy devices as part of an ASC, the binder-free rGO-PEDOT:PSS composite possesses the advantages of low cost, easy processability, scalable preparation, and greater environmental compatibility than polyaniline or polypyrrole, and exhibits excellent energy storage performance. In the context of the present study, it is believed that the present convenient and largescale method suggests an outstanding and practical way to prepare high-performance and low-cost composite electrode materials for energy storage device applications and a new route to binder-free electrode assembly.

\section{ACKNOWLEDGMENTS}

The authors are grateful to the Commonwealth of Australia, Automotive CRC 2020 and Australian Research Council (ARC) LIFE for financial support and Institute for Superconducting and Electronic Materials (ISEM) for use of its infrastructure as part of in-kind support. The authors would also like to thank Dr. Rouhollah Jalili for his kind technical assistance.

\section{SUPPLEMENTARY MATERIAL}

The Supplementary Material for this article can be found online at http://www.frontiersin.org/Journal/10.3389/fenrg.2014.00031/ abstract

\section{REFERENCES}

Aboutalebi, S. H., Chidembo, A. T., Salari, M., Konstantinov, K., Wexler, D., Liu, H. K., et al. (2011a). Comparison of GO, GO/MWCNTs composite and MWCNTs as potential electrode materials for supercapacitors. Energy Environ. Sci. 4, 1855-1865. doi:10.1039/clee01039e

Aboutalebi, S. H., Gudarzi, M. M., Zheng, Q. B., and Kim, J.-K. (2011b). Spontaneous formation of liquid crystals in ultralarge graphene oxide dispersions. $A d v$. Funct. Mater. 21, 2978-2988. doi:10.1002/adfm.201100448

Aboutalebi, S. H., Jalili, R., Esrafilzadeh, D., Salari, M., Gholamvand, Z., Aminorroaya Yamini, S., et al. (2014a). High-performance multifunctional graphene yarns: toward wearable all-carbon energy storage textiles. ACS Nano 8, 2456-2466. doi:10.1021/nn406026z

Aboutalebi, S. H., Jalili, R., Esrafilzadeh, D., Salari, M., Gholamvand, Z., Aminorroaya Yamini, S., et al. (2014b). High-performance multifunctional graphene yarns: toward wearable all-carbon energy storage textiles. ACS Nano 8, 2456-2466. doi:10.1021/nn406026z

Antiohos, D., Folkes, G., Sherrell, P., Ashraf, S., Wallace, G. G., Aitchison, P., et al. (2011). Compositional effects of PEDOT-PSS/single walled carbon nanotube films on supercapacitor device performance. J. Mater. Chem. 21, 15987-15994. doi:10.1039/cljm12986d

Bai, H., Li, C., and Shi, G. (2011). Functional composite materials based on chemically converted graphene. Adv. Mater. Weinheim 23, 1089-1115. doi:10.1002/ adma. 201003753

Chen, J., Li, C., and Shi, G. (2013). Graphene materials for electrochemical capacitors. J. Phys. Chem. Lett. 4, 1244-1253. doi:10.1021/jz400160k

Chen, Z., Ren, W., Gao, L., Liu, B., Pei, S., and Cheng, H.-M. (2011). Threedimensional flexible and conductive interconnected graphene networks grown by chemical vapour deposition. Nat. Mater. 10, 424-428. doi:10.1038/nmat3001

Chidembo, A. T., Aboutalebi, S. H., Konstantinov, K., Wexler, D., Liu, H. K., and Dou, S. X. (2013). Liquid crystalline dispersions of graphene-oxide-based hybrids: a practical approach towards the next generation of 3D isotropic architectures for energy storage applications. Part. Part. Syst. Charact. 31, 465-473. doi:10.1002/ppsc.201300254 
Choi, B. G., Yang, M., Hong, W. H., Choi, J. W., and Huh, Y. S. (2012). 3D macroporous graphene frameworks for supercapacitors with high energy and power densities. ACS Nano 6, 4020-4028. doi:10.1021/nn3003345

Fan, L., Zhang, N., and Sun, K. (2014). Flexible patterned micro-electrochemical capacitors based on PEDOT. Chem. Commun. (Camb.) 50, 6789-6792. doi:10. 1039/c4cc02280g

Fernández-Merino, M. J., Guardia, L., Paredes, J. I., Villar-Rodil, S., Soli'sFerna'ndez, P., Marti'nez-Alonso, A., et al. (2010). Vitamin C is an ideal substitute for hydrazine in the reduction of graphene oxide suspensions. J. Phys. Chem. C 114, 6426-6432. doi:10.1021/jp100603h

Friedel, B., Keivanidis, P. E., Brenner, T. J. K., Abrusci, A., Mcneill, C. R., Friend, R. H., et al. (2009). Effects of layer thickness and annealing of PEDOT:PSS layers in organic photodetectors. Macromolecules 42, 6741-6747. doi:10.1021/ma901182u

Hao, L., Li, X., and Zhi, L. (2013). Carbonaceous electrode materials for supercapacitors. Adv. Mater. Weinheim 25, 3899-3904. doi:10.1002/adma.201301204

Jalili, R., Aboutalebi, S. H., Esrafilzadeh, D., Konstantinov, K., Moulton, S. E., Razal, J. M., et al. (2013). Organic solvent-based graphene oxide liquid crystals: a facile route toward the next generation of self-assembled layer-by-layer multifunctional 3D architectures. ACS Nano 7, 3981-3990. doi:10.1021/nn305906z

Jo, K., Lee, T., Choi, H. J., Park, J. H., Lee, D. J., Lee, D. W., et al. (2011). Stable aqueous dispersion of reduced graphene nanosheets via non-covalent functionalization with conducting polymers and application in transparent electrodes. Langmuir 27, 2014-2018. doi:10.1021/la104420p

Kim, G. H., Hwang, D. H., and Woo, S. I. (2012). Thermoelectric properties of nanocomposite thin films prepared with poly(3,4-ethylenedioxythiophene) poly(styrenesulfonate) and graphene. Phys. Chem. Chem. Phys. 14, 3530-3536. doi:10.1039/c2cp23517j

Kim, J. E., Han, T. H., Lee, S. H., Kim, J. Y., Ahn, C. W., Yun, J. M., et al. (2011). Graphene oxide liquid crystals. Angew. Chem. Int. Ed. 50, 3043-3047. doi:10.1002/anie.201004692

Kuilla, T., Bhadra, S., Yao, D., Kim, N. H., Bose, S., and Lee, J. H. (2010). Recent advances in graphene based polymer composites. Prog. Polym. Sci. 35, 1350-1375. doi:10.1016/j.progpolymsci.2010.07.005

Lee, J. A., Shin, M. K., Kim, S. H., Cho, H. U., Spinks, G. M., Wallace, G. G., et al. (2013). Ultrafast charge and discharge biscrolled yarn supercapacitors for textiles and microdevices. Nat. Commun. 4, 1970. doi:10.1038/ncomms2970

Li, Z.-F., Zhang, H., Liu, Q., Sun, L., Stanciu, L., and Xie, J. (2013). Fabrication of high-surface-area graphene/polyaniline nanocomposites and their application in supercapacitors. ACS Appl. Mater. Interfaces 5, 2685-2691. doi:10.1021/ am4001634

Liu, C., Yu, Z., Neff, D., Zhamu, A., and Jang, B. Z. (2010). Graphene-based supercapacitor with an ultrahigh energy density. Nano Lett. 10, 4863-4868. doi: $10.1021 / \mathrm{nl} 102661 \mathrm{q}$

Liu, F., Song, S., Xue, D., and Zhang, H. (2012). Folded structured graphene paper for high performance electrode materials. Adv. Mater. Weinheim 24, 1089-1094. doi:10.1002/adma.201104691

Maiti, U. N., Lee, W. J., Lee, J. M., Oh, Y., Kim, J. Y., Kim, J. E., et al. (2014). 25th Anniversary article: chemically modified/doped carbon nanotubes \& graphene for optimized nanostructures \& nanodevices. Adv. Mater. Weinheim 26, 40-67. doi:10.1002/adma.201303265

Ouyang, J., Xu, Q., Chu, C.-W., Yang, Y., Li, G., and Shinar, J. (2004). On the mechanism of conductivity enhancement in poly(3,4ethylenedioxythiophene):poly(styrene sulfonate) film through solvent treatment. Polymer 45, 8443-8450. doi:10.1016/j.polymer.2004.10.001

Shen, T.-Z., Hong, S.-H., and Song, J.-K. (2014). Electro-optical switching of graphene oxide liquid crystals with an extremely large Kerr coefficient. Nat. Mater. 13, 394-399. doi:10.1038/nmat3888

Simon, P., and Gogotsi, Y. (2008). Materials for electrochemical capacitors. Nat. Mater. 7, 845-854. doi:10.1038/nmat2297

Snook, G. A., Kao, P., and Best, A. S. (2011). Conducting-polymer-based supercapacitor devices and electrodes. J. Power Sources 196, 1-12. doi:10.1016/j.jpowsour. 2010.06.084
Stoller, M. D., and Ruoff, R. S. (2010). Best practice methods for determining an electrode material's performance for ultracapacitors. Energy Environ. Sci. 3, 1294-1301. doi:10.1039/c0ee00074d

Sun, D., Jin, L., Chen, Y., Zhang, J.-R., and Zhu, J.-J. (2013). Microwave-assisted in situ synthesis of graphene/PEDOT hybrid and its application in supercapacitors. Chempluschem 78, 227-234. doi:10.1002/cplu.201200206

Sun, Y., and Shi, G. (2013). Graphene/polymer composites for energy applications. J. Polym. Sci. B Polym. Phys. 51, 231-253. doi:10.1002/polb.23226

Tan, Y. B., and Lee, J.-M. (2013). Graphene for supercapacitor applications. J. Mater. Chem. A 1, 14814-14843. doi:10.1039/c3ta12193c

Tang, P., Zhao, Y., Xu, C., and Ni, K. (2013). Enhanced energy density of asymmetric supercapacitors via optimizing negative electrode material and mass ratio of negative/positive electrodes. J. Sol. St. Electrochem. 17, 1701-1710. doi:10.1007/s10008-013-2021-7

Wang, G., Zhang, L., and Zhang, J. (2012). A review of electrode materials for electrochemical supercapacitors. Chem. Soc. Rev. 41, 797-828. doi:10.1039/ clcs15060j

Weng, Y.-T., and Wu, N.-L. (2013). High-performance poly(3,4-ethylenedioxythiophene):polystyrenesulfonate conducting-polymer supercapacitor containing hetero-dimensional carbon additives. J. Power Sources 238, 69-73. doi:10.1016/j.jpowsour.2013.03.070

Wu, Z.-S., Zhou, G., Yin, L.-C., Ren, W., Li, F., and Cheng, H.-M. (2012). Graphene/metal oxide composite electrode materials for energy storage. Nano Energy 1, 107-131. doi:10.1016/j.nanoen.2011.11.001

Xu, C., Xu, B., Gu, Y., Xiong, Z., Sun, J., and Zhao, X. S. (2013a). Graphene-based electrodes for electrochemical energy storage. Energy Environ. Sci. 6, 1388-1414. doi:10.1039/c3ee23870a

Xu, Y., Lin, Z., Huang, X., Liu, Y., Huang, Y., and Duan, X. (2013b). Flexible solidstate supercapacitors based on three-dimensional graphene hydrogel films. ACS Nano 7, 4042-4049. doi:10.1021/nn4000836

Yoo, D., Kim, J., and Kim, J. (2014). Direct synthesis of highly conductive poly(3,4ethylenedioxythiophene):poly(4-styrenesulfonate) (PEDOT:PSS)/graphene composites and their applications in energy harvesting systems. Nano Res. 7, 717-730. doi:10.1007/s12274-014-0433-z

Zhang, J., Jiang, J., Li, H., and Zhao, X. S. (2011). A high-performance asymmetric supercapacitor fabricated with graphene-based electrodes. Energy Environ. Sci. 4, 4009-4015. doi:10.1039/clee01354h

Zhang, J., Yang, H., Shen, G., Cheng, P., Zhang, J., and Guo, S. (2010). Reduction of graphene oxide vial-ascorbic acid. Chem. Commun. 46, 1112-1114. doi:10.1039/b917705a

Zhang, J., and Zhao, X. S. (2012). Conducting polymers directly coated on reduced graphene oxide sheets as high-performance supercapacitor electrodes. J. Phys. Chem. C 116, 5420-5426. doi:10.1021/jp211474e

Conflict of Interest Statement: The authors declare that the research was conducted in the absence of any commercial or financial relationships that could be construed as a potential conflict of interest.

Received: 16 June 2014; accepted: 19 July 2014; published online: 04 August 2014. Citation: Islam MM, Chidembo AT, Aboutalebi SH, Cardillo D, Liu HK, Konstantinov $K$ and Dou SX (2014) Liquid crystalline graphene oxide/PEDOT:PSS self-assembled $3 D$ architecture for binder-free supercapacitor electrodes. Front. Energy Res. 2:31. doi: 10.3389/fenrg.2014.00031

This article was submitted to Energy Storage, a section of the journal Frontiers in Energy Research.

Copyright (C) 2014 Islam, Chidembo, Aboutalebi, Cardillo, Liu, Konstantinov and Dou. This is an open-access article distributed under the terms of the Creative Commons Attribution License (CC BY). The use, distribution or reproduction in other forums is permitted, provided the original author(s) or licensor are credited and that the original publication in this journal is cited, in accordance with accepted academic practice. No use, distribution or reproduction is permitted which does not comply with these terms. 